\title{
Strong and Total Lagrange Dualities for Quasiconvex Programming
}

\author{
Donghui Fang, ${ }^{1}$ XianFa Luo, ${ }^{2}$ and Xianyun Wang ${ }^{1}$ \\ ${ }^{1}$ College of Mathematics and Statistics, Jishou University, Jishou 416000, China \\ ${ }^{2}$ Department of Mathematics, China Jiliang University, Hangzhou 310018, China
}

Correspondence should be addressed to Donghui Fang; dh_fang@163.com

Received 17 January 2014; Accepted 1 May 2014; Published 22 May 2014

Academic Editor: Ching-Jong Liao

Copyright (c) 2014 Donghui Fang et al. This is an open access article distributed under the Creative Commons Attribution License, which permits unrestricted use, distribution, and reproduction in any medium, provided the original work is properly cited.

\begin{abstract}
We consider the strong and total Lagrange dualities for infinite quasiconvex optimization problems. By using the epigraphs of the $z$ quasi-conjugates and the Greenberg-Pierskalla subdifferential of these functions, we introduce some new constraint qualifications. Under the new constraint qualifications, we provide some necessary and sufficient conditions for infinite quasiconvex optimization problems to have the strong and total Lagrange dualities.
\end{abstract}

\section{Introduction}

Consider the following infinite optimization problem:

$$
\begin{array}{ll}
\text { Minimize } & f(x), \\
\text { s.t. } & f_{t}(x) \leq 0, t \in T, \\
& x \in C,
\end{array}
$$

where $T$ is an arbitrary (possibly infinite) index set, $C$ is a nonempty convex subset of a locally convex (Hausdorff topological vector) space $X$, and $f, f_{t}: X \rightarrow \overline{\mathbb{R}}:=$ $\mathbb{R} \cup\{+\infty\}, t \in T$, are proper functions. This problem has been studied extensively under various degrees of restrictions imposed on the involved functions or on the underlying space and many problems in optimization and approximation theory such as linear semi-infinite optimization and the best approximation with restricted ranges can be recast into the form (1); see, for example, [1-12].

Observe that most works in the literature mentioned above were done under the assumptions that the involved functions are convex. Indeed, in mathematical programming, many of the problems naturally involve nonconvex functions. Recently, the quasiconvex programming, for which the involved functions are quasiconvex, has received much attention (cf. [13-18] and the references therein). Inspired by the works mentioned above, we continue to study the optimization problem (1) but with $f$ and $f_{t}$ being quasiconvex functions. The present paper is centered around the strong Lagrange duality and the total Lagrange duality for this quasiconvex programming. Usually for the strong Lagrange duality, one finds conditions ensuring the following equality:

$$
\inf _{x \in A} f(x)=\max _{\lambda \in \mathbb{R}_{+}^{(T)}} \inf _{x \in C}\left\{f(x)+\sum_{t \in T} \lambda_{t} f_{t}(x)\right\},
$$

and for the total Lagrange duality, one seeks conditions ensuring that the following implication holds for $x_{0} \in A:=$ $\left\{x \in C: f_{t}(x) \leq 0, \forall t \in T\right\}:$

$$
\begin{aligned}
{\left[f\left(x_{0}\right)\right.} & \left.=\min _{x \in A} f(x)\right] \\
\Longrightarrow & {\left[\exists \lambda \in \mathbb{R}_{+}^{(T)} \text {, s.t. } \inf _{x \in C}\left\{f(x)+\sum_{t \in T} \lambda_{t} f_{t}(x)\right\}\right.} \\
& \left.=f\left(x_{0}\right)\right],
\end{aligned}
$$

where

$$
\begin{aligned}
\mathbb{R}_{+}^{(T)}:= & \left\{\lambda=\left(\lambda_{t}\right) \in \mathbb{R}^{T}: \lambda_{t} \geq 0 \text { for each } t \in T\right. \\
& \text { and only finitely many } \left.\lambda_{t} \neq 0\right\} .
\end{aligned}
$$


To our knowledge, not many results are known to provide complete characterizations for the strong and total Lagrange dualities for quasiconvex programming.

Constraint qualifications involving epigraphs of the conjugate functions have been studied extensively. Our main aim in the present paper is to use these constraint qualifications (or their variations) to provide complete characterizations for the strong Lagrange duality and for the total Lagrange duality. It is well known that the Fenchel conjugate provides dual problems of convex minimization problems. In a similar way, different notions of conjugate for quasiconvex functions can be introduced in order to obtain dual problems of quasiconvex minimization problems. Note that the $z$-quasi-conjugate $(z \in \mathbb{R})$, defined by Greenberg and Pierskalla [13], plays in quasiconvex optimization the same role as the one Fenchel conjugate plays in convex optimization. Thus, by using the $z$ quasi-conjugate, we introduce a new constraint qualification which completely characterizes the strong Lagrange duality. Furthermore, many authors introduced some constraint qualifications involving the subdifferentials to establish the total Lagrange duality for convex programming. Similar to the convex case, we introduce the Greenberg-Pierskalla subdifferential to consider the total Lagrange duality for the quasiconvex programming.

The paper is organized as follows. The next section contains the necessary notations and preliminary results. In Section 3, some new constraint qualifications are provided and some relationships among them are given. In Section 4, we provide characterizations for the quasiconvex programming to have the strong Lagrange duality and the total Lagrange duality.

\section{Notations and Preliminary Results}

The notations used in this paper are standard (cf. [19]). In particular, we assume throughout the whole paper that $X$ is a real locally convex space and let $X^{*}$ denote the dual space of $X$. For $x \in X$ and $x^{*} \in X^{*}$, we write $\left\langle x^{*}, x\right\rangle$ for the value of $x^{*}$ at $x$; that is, $\left\langle x^{*}, x\right\rangle:=x^{*}(x)$. Let $Z$ be a set in $X$. The indicator function $\delta_{Z}$ of $Z$ is defined by

$$
\delta_{Z}(x):= \begin{cases}0, & x \in Z \\ +\infty, & \text { otherwise }\end{cases}
$$

The normal cone of $Z$ at $z_{0} \in Z$ is denoted by $N_{Z}\left(z_{0}\right)$ and is defined by

$$
N_{Z}\left(z_{0}\right):=\left\{x^{*} \in X^{*}:\left\langle x^{*}, z-z_{0}\right\rangle \leq 0 \forall z \in Z\right\} .
$$

Following [2], we use $\mathbb{R}^{(T)}$ to denote the space of real tuples $\lambda=\left(\lambda_{t}\right)_{t \in T}$ with only finitely many $\lambda_{t} \neq 0$, and let $\mathbb{R}_{+}^{(T)}$ denote the nonnegative cone in $\mathbb{R}^{(T)}$; that is,

$$
\mathbb{R}_{+}^{(T)}:=\left\{\left(\lambda_{t}\right)_{t \in T} \in \mathbb{R}^{(T)}: \lambda_{t} \geq 0 \text { for each } t \in T\right\} .
$$

Let $f: X \rightarrow \overline{\mathbb{R}}$ be a proper function. The effective domain, convex conjugate function, and epigraph of $f$ are denoted by $\operatorname{dom} f, f^{*}$, and epi $f$, respectively; they are defined by

$$
\begin{gathered}
\operatorname{dom} f:=\{x \in X: f(x)<+\infty\}, \\
f^{*}\left(x^{*}\right):=\sup \left\{\left\langle x^{*}, x\right\rangle-f(x): x \in X\right\} \\
\text { for each } x^{*} \in X^{*}, \\
\text { epi } f:=\{(x, r) \in X \times \mathbb{R}: f(x) \leq r\} .
\end{gathered}
$$

Recall that a function $f$ is said to be quasiconvex if, for all $x, y \in \mathbb{R}$ and $\alpha \in(0,1)$, the following inequality holds:

$$
f(\alpha x+(1-\alpha) y) \leq \max \{f(x), f(y)\}
$$

or equivalently its sublevel sets

$$
\{x \in X: f(x) \leq r\} \quad \text { for each } r \in \mathbb{R}
$$

are convex. Obviously, each convex function is quasiconvex. The following definition is taken from [13].

Definition 1. Let $z \in \mathbb{R}$. The $z$-quasi-conjugate of $f$ is a function $f_{z}^{*}: X^{*} \rightarrow \overline{\mathbb{R}}$ defined by

$$
f_{z}^{*}\left(x^{*}\right)=z-\inf \left\{f(y):\left\langle x^{*}, y\right\rangle \geq z\right\} .
$$

Note that (11) implies that

$$
f_{z}^{*}\left(x^{*}\right) \geq z-f(y) \quad \text { for each } y \in\left\{y \in X:\left\langle x^{*}, y\right\rangle \geq z\right\} \text {. }
$$

Then the $z$-quasi-conjugate function $f_{z}^{*}$ provides a lower bound for the corresponding conjugate function $f^{*}$ and, indeed, the conjugate function $f^{*}$ is the supremum of the $z$ quasi-conjugates $f_{z}^{*}$ over $z$. Moreover, by the definition, one finds that $f_{z}^{*}$ is quasiconvex for each $z \in \mathbb{R}$; that is,

$$
\begin{array}{r}
f_{z}^{*}\left(\alpha x_{1}^{*}+(1-\alpha) x_{2}^{*}\right) \leq \max \left\{f_{z}^{*}\left(x_{1}^{*}\right), f_{z}^{*}\left(x_{2}^{*}\right)\right\} \\
\text { for each } x_{1}^{*}, x_{2}^{*} \in X^{*}, \alpha \in(0,1) .
\end{array}
$$

For quasiconvex functions, several types of subdifferentials have been defined and observed by many researchers, for example, GP-subdifferential [13], $\mathbb{R}$-quasi-subdifferential [20], MLS-subdifferential [21], and so on. The classical Greenberg-Pierskalla subdifferential is among the simplest concepts, which is given as follows (cf. [13]).

Definition 2. The Greenberg-Pierskalla subdifferential of $f$ at $x \in \operatorname{dom} f$ is defined by

$$
\partial^{*} f(x):=\left\{x^{*} \in X^{*}: f(x)+f_{\left\langle x^{*}, x\right\rangle}^{*}\left(x^{*}\right)=\left\langle x^{*}, x\right\rangle\right\} .
$$

We also define

$$
\operatorname{dom} \partial^{*} f:=\left\{x \in X: \partial^{*} f(x) \neq \emptyset\right\}
$$

$\operatorname{im} \partial^{*} f:=\left\{x^{*} \in X^{*}: x^{*} \in \partial^{*} f(x)\right.$ for some $\left.x \in X\right\}$.

By definition,

$$
\partial^{*} f(x)=\left\{x^{*} \in X^{*}:\left\langle x^{*}, y-x\right\rangle \geq 0 \Longrightarrow f(y) \geq f(x)\right\},
$$


which is equivalent to the following equivalence, holds:

$$
x^{*} \in \partial^{*} f(x) \Longleftrightarrow\left\langle x^{*}, y-x\right\rangle<0 \text { for each } y \in S_{f}(x),
$$

where $S_{f}(x)$ denotes the strict sublevel sets

$$
S_{f}(x):=\{y \in X: f(y)<f(x)\} .
$$

Moreover, the following equivalence holds:

$$
0 \in \partial^{*} f(x) \Longleftrightarrow x \text { is a mimimizer of } f \text { on } X \text {. }
$$

Recall that the subdifferential of function $g$ at $x \in \operatorname{dom} g$ is defined by

$$
\begin{gathered}
\partial g(x):=\left\{x^{*} \in X^{*}: g(x)+\left\langle x^{*}, y-x\right\rangle\right. \\
\leq g(y) \text { for each } y \in X\} .
\end{gathered}
$$

Then,

$$
\partial g(x) \subseteq \partial^{*} g(x) \text { for each } x \in \operatorname{dom} g
$$

By definition, we can obtain the following lemma easily, which was proved in [13] when $X=\mathbb{R}^{n}$.

Lemma 3. Let $g$, $h$ be proper quasiconvex functions on $X$ and let $x_{0} \in \operatorname{dom} g \cap \operatorname{dom} h$. Then the following statements hold.

(i) If $g \leq h$, then $g_{z}^{*} \geq h_{z}^{*}$ and hence epi $g_{z}^{*} \subseteq$ epi $h_{z}^{*}$ for each $z \in \mathbb{R}$.

(ii) If $g \leq h$ and $g\left(x_{0}\right)=h\left(x_{0}\right)$, then $\partial^{*} g\left(x_{0}\right) \subseteq \partial^{*} h\left(x_{0}\right)$.

The following lemma is known in [19, Theorem 2.8.7].

Lemma 4. Let $g, h: X \rightarrow \overline{\mathbb{R}}$ be proper convex functions. If $g$ or $h$ is continuous at some point of $\operatorname{dom} g \cap \operatorname{dom} h$, then

$$
\begin{aligned}
& \text { epi }(g+h)^{*}=\text { epi } g^{*}+\text { epi } h^{*}, \\
& \partial(g+h)(x)=\partial g(x)+\partial h(x)
\end{aligned}
$$

for each $x \in \operatorname{dom} g \cap \operatorname{dom} h$;

consequently, if $h \in X^{*}$, then

$$
\begin{gathered}
\text { epi }(g+h)^{*}=\text { epi } g^{*}+\{h\} \times[0,+\infty), \\
\partial(g+h)(x)=\partial g(x)+h \quad \text { for each } x \in \operatorname{dom} g .
\end{gathered}
$$

The following example shows that (22) do not necessarily hold if $g$ is a quasiconvex function even in the case when $h \in$ $X^{*}$.

Example 5. Let $X:=\mathbb{R}$ and define the function $g: \mathbb{R} \rightarrow \mathbb{R}$ by

$$
g(x):= \begin{cases}1, & x \geq 0 \\ 0, & x<0\end{cases}
$$

Then

$$
\begin{gathered}
\text { epi } g_{0}^{*}=((0,+\infty) \times[-1,+\infty)) \cup((-\infty, 0] \times[0,+\infty)), \\
\partial^{*} g(0)=\left\{x^{*} \in \mathbb{R}: x^{*} \cdot x<0 \text { for each } x<0\right\}=(0,+\infty) .
\end{gathered}
$$

Take $p=2$. Then for each $x^{*} \in \mathbb{R}$,

$$
\begin{aligned}
(g+p)_{0}^{*}\left(x^{*}\right) & = \begin{cases}-1, & x^{*}>0 \\
+\infty, & x^{*} \leq 0\end{cases} \\
p_{0}^{*}\left(x^{*}\right) & = \begin{cases}+\infty, & x^{*}>0, \\
0, & x^{*} \leq 0,\end{cases}
\end{aligned}
$$

and hence

$$
\begin{gathered}
\text { epi }(g+p)_{0}^{*}=(0,+\infty) \times[-1,+\infty), \\
\text { epi } p_{0}^{*}=(-\infty, 0] \times[0,+\infty),
\end{gathered}
$$

Therefore,

$$
\text { epi }(g+p)_{0}^{*} \neq \text { epi } g_{0}^{*}+\text { epi } p_{0}^{*} \text {. }
$$

On the other hand, take $p=-2$. Then $S_{g+p}(0)=(-\infty, 0)$ and $S_{p}(0)=\emptyset$. Hence,

$$
\begin{gathered}
\partial^{*}(g+p)(0)=\left\{x^{*} \in \mathbb{R}: x^{*} \cdot x<0\right. \\
\text { for each } \left.x \in S_{g+p}(0)\right\}=(0,+\infty), \\
\partial^{*} p(0)=\left\{x^{*} \in \mathbb{R}: x^{*} \cdot x<0 \text { for each } x \in S_{p}(0)\right\}=\mathbb{R} .
\end{gathered}
$$

Therefore,

$$
\partial^{*}(g+p)(0) \neq \partial^{*} g(0)+\partial^{*} p(0)
$$

\section{New Regularity Conditions for Lagrange Dualities}

Unless explicitly stated otherwise, let $T, C, f,\left\{f_{t}: t \in T\right\}$ and $A$ be as in Section 1; namely, $T$ is an index set, $C \subseteq X$ is a convex set, $f$ and $f_{t}, t \in T$ are proper quasiconvex functions such that $f+\delta_{C}+\sum_{t \in T} \lambda_{t} f_{t}$ is quasiconvex for each $\lambda \in \mathbb{R}_{+}^{(T)}$, and $A \neq \emptyset$ is the solution set of the following system:

$$
x \in C ; \quad f_{t}(x) \leq 0 \quad \text { for each } t \in T \text {. }
$$

Then, $A$ is a convex set. Throughout we also assume that dom $f \cap A \neq \emptyset$. For each $x \in X$, let $T(x)$ be the active index set of system (31); that is,

$$
T(x):=\left\{t \in T: f_{t}(x)=0\right\} .
$$

To study the strong Lagrange duality and the total Lagrange duality, we need the following regularity conditions. 
Definition 6. The family $\left\{\delta_{C} ; f_{t}: t \in T\right\}$ is said to have

(a) the quasi-(WEHP) if

epi $\left(f+\delta_{A}\right)_{0}^{*} \cap(\{0\} \times \mathbb{R})$

$$
=\bigcup_{\lambda \in \mathbb{R}_{+}^{(T)}} \operatorname{epi}\left(f+\delta_{C}+\sum_{t \in T} \lambda_{t} f_{t}\right)_{0}^{*} \cap(\{0\} \times \mathbb{R}) ;
$$

(b) the quasi-(WBCQ) at $x \in \operatorname{dom} f \cap A$ if

$$
\begin{aligned}
\partial^{*}\left(f+\delta_{A}\right)(x) \cap\{0\} & =\bigcup_{\substack{\lambda \in \mathbb{R}_{+}^{(T)} \\
\sum_{t \in T} \lambda_{t} f_{t}(x)=0}} \partial^{*}\left(f+\delta_{C}+\sum_{t \in T} \lambda_{t} f_{t}\right)(x) \cap\{0\} ;
\end{aligned}
$$

(c) the quasi-(WBCQ) if it has the quasi-(WBCQ) at each point in $\operatorname{dom} f \cap A$.

Remark 7. Note that $f+\delta_{C}+\sum_{t \in T} \lambda_{t} f_{t} \leq f+\delta_{A}$ holds for each $\lambda \in \mathbb{R}_{+}^{(T)}$. Then, by Lemma $3(\mathrm{i}),\left(f+\delta_{C}+\sum_{t \in T} \lambda_{t} f_{t}\right)_{z}^{*} \geq$ $\left(f+\delta_{A}\right)_{z}^{*}$ for each $z \in \mathbb{R}$ and hence

$$
\bigcup_{\lambda \in \mathbb{R}_{+}^{(T)}} \operatorname{epi}\left(f+\delta_{C}+\sum_{t \in T} \lambda_{t} f_{t}\right)_{z}^{*} \subseteq \operatorname{epi}\left(f+\delta_{A}\right)_{z}^{*}
$$

for each $z \in \mathbb{R}$.

Moreover, for each $\lambda \in \mathbb{R}_{+}^{(T)}$ satisfying $\sum_{t \in T} \lambda_{t} f_{t}(x)=0$,

$$
f(x)+\delta_{A}(x)=f(x)=f(x)+\delta_{C}(x)+\sum_{t \in T} \lambda_{t} f_{t}(x)
$$

for each $x \in A$.

Thus, by Lemma 3(ii), we have

$$
\bigcup_{\substack{\lambda \in \mathbb{R}_{+}^{(T)} \\ \sum_{t \in T} \lambda_{t} f_{t}(x)=0}} \partial^{*}\left(f+\delta_{C}+\sum_{t \in T} \lambda_{t} f_{t}\right)(x) \subseteq \partial^{*}\left(f+\delta_{A}\right)(x) .
$$

Therefore, (33) holds if and only if

$$
\begin{aligned}
& \operatorname{epi}\left(f+\delta_{A}\right)_{0}^{*} \cap(\{0\} \times \mathbb{R}) \\
& \quad \subseteq \bigcup_{\lambda \in \mathbb{R}_{+}^{(T)}} \operatorname{epi}\left(f+\delta_{C}+\sum_{t \in T} \lambda_{t} f_{t}\right)_{0}^{*} \cap(\{0\} \times \mathbb{R}) ;
\end{aligned}
$$

and (34) holds if and only if the following inclusion holds:

$$
\begin{aligned}
\partial^{*}\left(f+\delta_{A}\right)(x) \cap\{0\} \\
\subseteq \bigcup_{\substack{\lambda \in \mathbb{R}_{+}^{(T)} \\
\sum_{t \in T} \lambda_{t} f_{t}(x)=0}} \partial^{*}\left(f+\delta_{C}+\sum_{t \in T} \lambda_{t} f_{t}\right)(x) \cap\{0\} .
\end{aligned}
$$

The following proposition describes the relationship between the quasi-(WEHP) and the quasi-(WBCQ).
Proposition 8. The following implication holds:

$$
\text { the quasi- }(W E H P) \Longrightarrow \text { the quasi-(WBCQ). }
$$

Furthermore, if $0 \in \operatorname{im} \partial^{*}\left(f+\delta_{A}\right)$, then

$$
\text { the quasi- }(W E H P) \Longleftrightarrow \text { the quasi- (WBCQ). }
$$

Proof. Suppose that the quasi-(WEHP) holds. To show the quasi-(WBCQ), by Remark 7, it suffices to show (39) holds. To do this, let $x_{0} \in \operatorname{dom} f \cap A$ and let $0 \in \partial^{*}\left(f+\delta_{A}\right)\left(x_{0}\right)$. Then by (19), $f\left(x_{0}\right) \leq f(x)$ for each $x \in A$. Hence, by the definition of 0 -conjugate function,

$$
\begin{aligned}
\left(0,-f\left(x_{0}\right)\right) & \in \operatorname{epi}\left(f+\delta_{A}\right)_{0}^{*} \\
& =\bigcup_{\lambda \in \mathbb{R}_{+}^{(T)}} \operatorname{epi}\left(f+\delta_{C}+\sum_{t \in T} \lambda_{t} f_{t}\right)_{0}^{*},
\end{aligned}
$$

thanks to the assumed quasi-(WEHP). This implies that there exists $\lambda \in \mathbb{R}_{+}^{(T)}$ such that

$$
\left(0,-f\left(x_{0}\right)\right) \in \operatorname{epi}\left(f+\delta_{C}+\sum_{t \in J} \lambda_{t} f_{t}\right)_{0}^{*},
$$

where $J \subseteq T$ is a finite subset and $\left(\lambda_{t}\right) \in \mathbb{R}_{+}^{(T)}$ with $J=\{t \in$ $\left.T: \lambda_{t} \neq 0\right\}$. By definition, (43) is equivalent to

$$
\left(f+\delta_{\mathrm{C}}+\sum_{t \in J} \lambda_{t} f_{t}\right)(x) \geq f\left(x_{0}\right) \geq\left(f+\delta_{\mathrm{C}}+\sum_{t \in J} \lambda_{t} f_{t}\right)\left(x_{0}\right)
$$

for each $x \in X$,

where the last inequality holds because $f_{t}\left(x_{0}\right) \leq 0$ for each $t \in J$, while, by (19), (44) holds if and only if

$$
0 \in \partial^{*}\left(f+\delta_{C}+\sum_{t \in J} \lambda_{t} f_{t}\right)\left(x_{0}\right)
$$

Below we show that $J \subseteq T\left(x_{0}\right)$. Note by (43) that

$$
\left(f+\delta_{C}+\sum_{t \in J} \lambda_{t} f_{t}\right)_{0}^{*}(0) \leq-f\left(x_{0}\right)
$$

while, by definition,

$$
\left(f+\delta_{C}+\sum_{t \in J} \lambda_{t} f_{t}\right)_{0}^{*}(0) \geq-\left(f+\delta_{C}+\sum_{t \in J} \lambda_{t} f_{t}\right)\left(x_{0}\right) .
$$

Hence, by the above inequalities, one has that

$$
\left(f+\delta_{C}+\sum_{t \in J} \lambda_{t} f_{t}\right)\left(x_{0}\right) \geq f\left(x_{0}\right) .
$$


Since $\lambda_{t}>0$ and $f_{t}(x) \leq 0$ for each $t \in J$, this implies that $\lambda_{t} f_{t}(x)=0$; that is, $f_{t}(x)=0$ for each $t \in J$. Thus $J \subseteq T(x)$, and hence, (39) holds.

Conversely, suppose that $0 \in \operatorname{Im} \partial^{*}\left(f+\delta_{A}\right)$. To show the quasi-(WEHP), by Remark 7, we only need to show (38) holds. To do this, note that $0 \in \operatorname{Im} \partial^{*}\left(f+\delta_{A}\right)$. Then there exists $x_{0} \in \operatorname{dom} f \cap A$ such that

$$
\begin{aligned}
& 0 \in \partial^{*}\left(f+\delta_{A}\right)\left(x_{0}\right) \\
& \subseteq \bigcup_{\substack{\lambda \in \mathbb{R}_{+}^{(T)} \\
\sum_{t \in T} \lambda_{t} f_{t}\left(x_{0}\right)=0}} \partial^{*}\left(f+\delta_{C}+\sum_{t \in T} \lambda_{t} f_{t}\right)\left(x_{0}\right),
\end{aligned}
$$

thanks to the assumed quasi-(WBCQ). Therefore, there exists $\lambda \in \mathbb{R}_{+}^{(T)}$ such that

$$
x^{*} \in \partial^{*}\left(f+\delta_{C}+\sum_{t \in T} \lambda_{t} f_{t}\right)(0)
$$

with

$$
\sum_{t \in T} \lambda_{t} f_{t}\left(x_{0}\right)=0
$$

These two relations imply that

$$
f\left(x_{0}\right)+\left(f+\delta_{C}+\sum_{t \in T} \lambda_{t} f_{t}\right)_{0}^{*}(0)=0 .
$$

Moreover, since $0 \in \partial^{*}\left(f+\delta_{A}\right)\left(x_{0}\right)$, it follows that

$$
f\left(x_{0}\right)+\left(f+\delta_{A}\right)_{0}^{*}(0)=0 .
$$

Combining (53) with (52), we have that

$$
\left(f+\delta_{A}\right)_{0}^{*}(0)=\left(f+\delta_{C}+\sum_{t \in T} \lambda_{t} f_{t}\right)_{0}^{*}(0) .
$$

Hence, for each $r \in \mathbb{R}$ satisfying $(0, r) \in \operatorname{epi}\left(f+\delta_{A}\right)_{0}^{*}$, we have

$$
\begin{aligned}
(0, r) & \in \operatorname{epi}\left(f+\delta_{C}+\sum_{t \in T} \lambda_{t} f_{t}\right)_{0}^{*} \\
& \subseteq \bigcup_{\lambda \in \mathbb{R}_{+}^{(T)}} \operatorname{epi}\left(f+\delta_{C}+\sum_{t \in T} \lambda_{t} f_{t}\right)_{0}^{*} .
\end{aligned}
$$

Thus, (38) holds and the proof is complete.

\section{Strong and Total Lagrange Dualities for Infinite Quasiconvex Programming}

Consider the following quasiconvex programming:

$(P) \quad$ Minimize $f(x)$,

$$
\begin{array}{ll}
\text { s.t. } & f_{t}(x) \leq 0, t \in T, \\
& x \in C .
\end{array}
$$

Its dual problem is defined by

$$
\text { (D) } \sup _{\lambda \in \mathbb{R}_{+}^{(T)}} \inf _{x \in C}\left\{f(x)+\sum_{t \in T} \lambda_{t} f_{t}(x)\right\} .
$$

We denote by $v(P)$ and $v(D)$ the optimal objective values of $(P)$ and $(D)$, respectively. Clearly, $v(P) \geq v(D)$; that is, the weak Lagrange duality holds between $(P)$ and $(D)$. We say that the strong Lagrange duality between $(P)$ and $(D)$ holds if there is no duality gap (i.e., $v(P)=v(D)$ ) and the dual problem $(D)$ has an optimal solution. The following theorem gives some sufficient and necessary conditions to ensure that the strong Lagrange duality holds.

Theorem 9. The following statements are equivalent.

(i) For each $\alpha \in \mathbb{R}$,

$$
\begin{aligned}
& (0,-\alpha) \in \operatorname{epi}\left(f+\delta_{A}\right)_{0}^{*} \\
& \Longleftrightarrow\left[\exists\left(\lambda_{t}\right)_{t \in T} \in \mathbb{R}_{+}^{(T)} \text { s.t. }(0,-\alpha)\right. \\
& \left.\quad \in \operatorname{epi}\left(f+\delta_{C}+\sum_{t \in T} \lambda_{t} f_{t}\right)_{0}^{*}\right] .
\end{aligned}
$$

(ii) The family $\left\{\delta_{C} ; f_{t}: t \in T\right\}$ has the quasi-(WEHP).

(iii) The strong Lagrange duality holds between $(P)$ and (D).

Proof. It is evident that (i) $\Leftrightarrow$ (ii). Below we show that (i) $\Leftrightarrow$ (iii). To do this, note that, for each $\alpha \in \mathbb{R}$,

$$
\begin{gathered}
(0,-\alpha) \in \operatorname{epi}\left(f+\delta_{A}\right)_{0}^{*} \\
\Longleftrightarrow\left(f+\delta_{A}\right)_{0}^{*}(0) \leq-\alpha \\
\Longleftrightarrow f(x) \geq \alpha \quad \text { for each } x \in A, \\
(0,-\alpha) \in \bigcup_{\lambda \in \mathbb{R}_{+}^{(T)}} \operatorname{epi}\left(f+\delta_{C}+\sum_{t \in T} \lambda_{t} f_{t}\right)_{0}^{*} \\
\Longleftrightarrow \exists\left(\lambda_{t}\right)_{t \in T} \in \mathbb{R}_{+}^{(T)}, \\
\text { s.t. } f(x)+\sum_{t \in T} \lambda_{t} f_{t}(x) \geq \alpha \quad \text { for each } x \in C .
\end{gathered}
$$

Suppose that (i) holds. Let $r:=v(P) \in \mathbb{R}$ (if $v(P)=$ $-\infty$, then the result holds trivially). Then, by $(59),(0,-r) \in$ $\operatorname{epi}\left(f+\delta_{A}\right)_{0}^{*}$ and $(0,-r) \in \bigcup_{\lambda \in \mathbb{R}_{+}^{(T)}} \operatorname{epi}\left(f+\delta_{C}+\sum_{t \in T} \lambda_{t} f_{t}\right)_{0}^{*}$ by (i). Hence, using (59) and the definition of $v(D)$, we see that $v(D) \geq r$ and the problem $(D)$ has an optimal solution. This together with the weak Lagrange duality implies that the strong Lagrange duality holds.

Conversely, suppose that the strong Lagrange duality holds. To show (i), by Remark 7 , we only need to show that (38) holds. To do this, let $(0,-r) \in \operatorname{epi}\left(f+\delta_{A}\right)_{0}^{*}$. Then, by (59), $v(p) \geq r$ and $v(D) \geq r$ by (iii). Hence, by (59), we see that $(0,-r) \in \bigcup_{\lambda \in \mathbb{R}_{+}^{(T)}} \operatorname{epi}\left(f+\delta_{C}+\sum_{t \in T} \lambda_{t} f_{t}\right)_{0}^{*}$. Therefore, (38) is proved and the proof is complete. 
The following two examples illustrate Theorem 9.

Example 10. Let $X=C:=\mathbb{R}$ and let $T:=\{1\}$. Define the function $f, f_{1}: \mathbb{R} \rightarrow \mathbb{R}$ by

$$
f(x):=\left\{\begin{array}{ll}
1, & x \geq 0, \\
0, & x<0,
\end{array} \quad f_{1}(x):=x^{2}-1 \quad \text { for each } x \in \mathbb{R} .\right.
$$

Then $f$ is quasiconvex and $A=[-1,1]$. Note that, for each $x \in \mathbb{R}$ and each $\lambda \geq 0$,

$$
\begin{gathered}
\left(f+\delta_{A}\right)(x)= \begin{cases}1, & 0 \leq x \leq 1, \\
0, & -1 \leq x<0, \\
+\infty, & x>1 \text { or } x<-1,\end{cases} \\
\left(f+\delta_{C}+\lambda f_{1}\right)(x)= \begin{cases}1+\lambda\left(x^{2}-1\right), & x \geq 0, \\
\lambda\left(x^{2}-1\right), & x<0 .\end{cases}
\end{gathered}
$$

Then for each $x^{*} \in \mathbb{R}$, it is easy to see that

$$
\begin{aligned}
\left(f+\delta_{A}\right)_{0}^{*}\left(x^{*}\right) & =-\inf \left\{\left(f+\delta_{A}\right)(x): x^{*} x \geq 0\right\} \\
& = \begin{cases}-1, & x^{*}>0, \\
0, & x^{*} \leq 0,\end{cases}
\end{aligned}
$$

and for each $\lambda \geq 0$,

$$
\begin{aligned}
\left(f+\delta_{C}+\lambda f_{1}\right)_{0}^{*}\left(x^{*}\right) & =-\inf \left\{\left(f+\delta_{C}+\lambda f_{1}\right)(x): x^{*} x \geq 0\right\} \\
& = \begin{cases}-1+\lambda, & x^{*}>0, \\
\lambda, & x^{*} \leq 0 .\end{cases}
\end{aligned}
$$

Hence,

$$
\begin{aligned}
\operatorname{epi}\left(f+\delta_{A}\right)_{0}^{*} & =\bigcup_{\lambda \geq 0} \operatorname{epi}\left(f+\delta_{C}+\lambda f_{1}\right)_{0}^{*} \\
& =\left((-\infty, 0] \times \mathbb{R}_{+}\right) \cup((0,+\infty) \times[-1,+\infty)) .
\end{aligned}
$$

Therefore, by Theorem 9, we see that the strong Lagrange duality holds. In fact, $v(P)=v(D)=0$ and $\lambda=0$ is an optimal solution to $(D)$.

Example 11. Let $X=C:=\mathbb{R}$ and let $T:=\{1\}$. Define the function $f, f_{1}: \mathbb{R} \rightarrow \mathbb{R}$ by

$$
f(x):=\left\{\begin{array}{ll}
1, & x \geq 0, \\
0, & x<0,
\end{array} \quad f_{1}(x):=-x \quad \text { for each } x \in \mathbb{R} .\right.
$$

Then $f$ is quasiconvex and $A=[0,+\infty)$. Note that, for each $x \in \mathbb{R}$ and each $\lambda \geq 0$,

$$
\begin{gathered}
\left(f+\delta_{A}\right)(x)= \begin{cases}1, & x \geq 0, \\
+\infty, & x<0,\end{cases} \\
\left(f+\delta_{C}+\lambda f_{1}\right)(x)= \begin{cases}1-\lambda x, & x \geq 0, \\
-\lambda x, & x<0 .\end{cases}
\end{gathered}
$$

Then for each $x^{*} \in \mathbb{R}$, it is easy to see that

$$
\left(f+\delta_{A}\right)_{0}^{*}\left(x^{*}\right)=-\inf \left\{\left(f+\delta_{A}\right)(x): x^{*} x \geq 0\right\}=-1,
$$

and for each $\lambda \geq 0$,

$$
\begin{aligned}
\left(f+\delta_{C}+\lambda f_{1}\right)_{0}^{*}\left(x^{*}\right) & =-\inf \left\{\left(f+\delta_{C}+\lambda f_{1}\right)(x): x^{*} x \geq 0\right\} \\
& = \begin{cases}-1, & x^{*}>0, \lambda=0, \\
+\infty, & x^{*}>0, \lambda>0, \\
0, & x^{*} \leq 0 .\end{cases}
\end{aligned}
$$

Hence,

$$
\begin{gathered}
\operatorname{epi}\left(f+\delta_{A}\right)_{0}^{*}=\mathbb{R} \times[-1,+\infty), \\
\bigcup_{\lambda \geq 0} \operatorname{epi}\left(f+\delta_{C}+\lambda f_{1}\right)_{0}^{*} \\
=((0,+\infty) \times[-1,+\infty)) \cup((-\infty, 0] \times[0,+\infty)) .
\end{gathered}
$$

Therefore, the quasi-(WEHP) does not hold and, by Theorem 9, the strong Lagrange duality does not hold (in fact, $v(P)=1$ and $v(D)=-\infty)$.

Remark 12. (a) In [5, Theorem 5.1], the authors showed that the strong Lagrange duality holds between $(P)$ and $(D)$ if and only if the following condition holds:

(iv) $\operatorname{epi}\left(f+\delta_{A}\right)^{*} \cap(\{0\} \times \mathbb{R})$

$$
=\bigcup_{\lambda \in \mathbb{R}_{+}^{(T)}} \operatorname{epi}\left(f+\delta_{C}+\sum_{t \in T} \lambda_{t} f_{t}\right)^{*} \cap(\{0\} \times \mathbb{R}) .
$$

Thus, by Theorem 9, the statements (i), (ii), and (iii) of Theorem 9 are equivalent to (iv).

(b) Recall from [5] that the stable strong Lagrange duality holds between $(P)$ and $(D)$ if, for each $p^{*} \in X^{*}$, the following equality holds:

$$
\begin{aligned}
\inf _{x \in A} & \{f(x)+\langle p, x\rangle\} \\
& =\max _{\lambda \in \mathbb{R}_{+}^{(T)}} \inf _{x \in C}\left\{f(x)+\langle p, x\rangle+\sum_{t \in T} \lambda_{t} f_{t}(x)\right\} .
\end{aligned}
$$

In [5], the authors show that the stable strong Lagrange duality holds if and and only if the family $\left\{\delta_{C} ; f_{t}: t \in T\right\}$ has the conical (WEHP) ${ }_{f}$; that is,

$$
\operatorname{epi}\left(f+\delta_{A}\right)^{*}=\bigcup_{\lambda \in \mathbb{R}_{+}^{(T)}} \operatorname{epi}\left(f+\delta_{C}+\sum_{t \in T} \lambda_{t} f_{t}\right)^{*}
$$

Naturally, we wonder if the equivalence still holds if we replace the convex conjugate function by the $z$-quasiconjugate function. However, the following example shows that the stable strong Lagrange duality is not equivalent to

$$
\operatorname{epi}\left(f+\delta_{A}\right)_{0}^{*}=\bigcup_{\lambda \in \mathbb{R}_{+}^{(T)}} \operatorname{epi}\left(f+\delta_{C}+\sum_{t \in T} \lambda_{t} f_{t}\right)_{0}^{*} .
$$


Example 13. Let $X=C:=\mathbb{R}$ and $T:=\{1\}$. Define $f, f_{1}$ : $\mathbb{R} \rightarrow \mathbb{R}$ as in Example 10. Then by (64), we see that (73) holds. However, it is easy to see that, for each $y \in \mathbb{R}$,

$$
\left(f+\delta_{A}\right)^{*}(y)= \begin{cases}y-1, & y \geq 1, \\ 0, & 0 \leq y<1, \\ -y, & y<0\end{cases}
$$

and, for each $\lambda>0$,

$$
\left(f+\delta_{C}+\lambda f_{1}\right)^{*}(y)=\frac{y^{2}}{4 \lambda}+\lambda .
$$

Thus,

$$
\begin{aligned}
& \operatorname{epi}\left(f+\delta_{A}\right)^{*} \\
&=\left\{(x, y) \in \mathbb{R}^{2}: x \geq 1, y \geq x-1\right\} \cup\left([0,1) \times \mathbb{R}_{+}\right) \\
& \cup\left\{(x, y) \in \mathbb{R}^{2}: x<0, y \geq-x\right\}, \\
& \bigcup_{\lambda \geq 0} \operatorname{epi}\left(f+\delta_{C}+\lambda f_{1}\right)^{*}=(\mathbb{R} \times(0,+\infty)) \cup\left(\{0\} \times \mathbb{R}_{+}\right) .
\end{aligned}
$$

This implies that

$$
\operatorname{epi}\left(f+\delta_{A}\right)^{*} \neq \bigcup_{\lambda \geq 0} \operatorname{epi}\left(f+\delta_{C}+\lambda f_{1}\right)^{*} .
$$

Hence, by [5, Theorem 5.2], the stable strong duality does not hold. Therefore, the stable strong Lagrange duality and (73) are not equivalent.

In the remainder of this section, we study the total Lagrange duality problem; that is, when does the strong duality hold between $(P)$ and $(D)$ (assuming that $\emptyset \neq S(P):=$ $\left.\left\{y \in A: f(x)=\min _{x \in A} f(x)\right\}\right)$ ? Obviously, if the strong duality holds between $(P)$ and $(D)$, then so does the total duality. Hence, if one of conditions in Theorem 9 holds, then the total duality holds. Below we give some sufficient and necessary conditions to ensure that the total duality holds.

Theorem 14. The following assertions are equivalent.

(i) The total Lagrange duality holds between $(P)$ and $(D)$.

(ii) For each $x \in \operatorname{dom} f \cap A$,

$$
\begin{aligned}
& 0 \in \partial^{*}\left(f+\delta_{A}\right)(x) \\
& \Longleftrightarrow 0 \in \bigcup_{\substack{\lambda \in \mathbb{R}_{+}^{(T)} \\
\sum_{t \in T} \lambda_{t} f_{t}(x)=0}} \partial^{*}\left(f+\delta_{C}+\sum_{t \in T} \lambda_{t} f_{t}\right)(x) .
\end{aligned}
$$

(iii) For each $x \in \operatorname{dom} f \cap A$,

$$
\begin{aligned}
\partial^{*} & \left(f+\delta_{A}\right)(x) \\
& =\bigcup_{\substack{\lambda \in \mathbb{R}_{+}^{(T)} \\
\sum_{t \in T} \lambda_{t} f_{t}(x)=0}} \partial^{*}\left(f+\delta_{C}+\sum_{t \in T} \lambda_{t} f_{t}\right)(x)=X^{*} .
\end{aligned}
$$

(iv) The family $\left\{\delta_{C} ; f_{t}: t \in T\right\}$ has the quasi-(WBCQ).
Proof. It is evident that (ii) $\Leftrightarrow$ (iv). Note that

$$
\begin{aligned}
& 0 \in \partial^{*}\left(f+\delta_{A}\right)(x) \Longleftrightarrow \partial^{*}\left(f+\delta_{A}\right)(x)=X^{*}, \\
& 0 \in \bigcup_{\substack{\lambda \in \mathbb{R}_{+}^{(T)} \\
\sum_{t \in T} \lambda_{t} f_{t}(x)=0}} \partial^{*}\left(f+\delta_{C}+\sum_{t \in T} \lambda_{t} f_{t}\right)(x) \\
& \Longleftrightarrow \bigcup_{\substack{\lambda \in \mathbb{R}^{(T)} \\
\sum_{t \in T} \lambda_{t} f_{t}(x)=0}} \partial^{*}\left(f+\delta_{C}+\sum_{t \in T} \lambda_{t} f_{t}\right)(x)=X^{*} .
\end{aligned}
$$

Hence, (ii) $\Leftrightarrow$ (iii). Suppose that (iv) holds. Let $f\left(x_{0}\right)=$ $\min _{x \in A} f(x)$. Then by (19), $0 \in \partial^{*}\left(f+\delta_{A}\right)\left(x_{0}\right)$. This implies that $0 \in \operatorname{Im} \partial^{*}\left(f+\delta_{A}\right)$. Hence, by Proposition 8 , the quasi-(WEHP) holds and by Theorem 9 , the strong Lagrange duality holds between $(P)$ and $(D)$. Therefore, (i) holds and the implication (iv) $\Rightarrow$ (i) is proved. Below we only need to show that (i) $\Rightarrow$ (iv). To do this, assume that (i) holds. Let $x_{0} \in \operatorname{dom} f \cap A$. To show the quasi-(WBCQ), it suffices by Remark 7 to show that (39) holds with $x_{0}$ in place of $x$. To do this, let $0 \in \partial^{*}\left(f+\delta_{A}\right)\left(x_{0}\right)$. Then by (19), $f\left(x_{0}\right)=$ $\min _{x \in A} f(x)$. Since the strong duality holds between $\left(P_{f}\right)$ and $(D)$, it follows that there exists $\lambda \in \mathbb{R}_{+}^{(T)}$ such that

$$
\begin{aligned}
f\left(x_{0}\right) & =\inf _{x \in C}\left\{f(x)+\sum_{t \in T} \lambda_{t} f_{t}(x)\right\} \\
& \leq f\left(x_{0}\right)+\sum_{t \in T} \lambda_{t} f_{t}\left(x_{0}\right) \leq f\left(x_{0}\right),
\end{aligned}
$$

where the last inequality holds because $x_{0} \in A$. Hence, $\sum_{t \in T} \lambda_{t} f_{t}\left(x_{0}\right)=0$ and it follows that

$$
\left(f+\delta_{C}+\sum_{t \in T} \lambda_{t} f_{t}\right)\left(x_{0}\right) \leq\left(f+\delta_{C}+\sum_{t \in T} \lambda_{t} f_{t}\right)(x)
$$

for each $x \in X$,

which by (19) implies that $0 \in \partial^{*}\left(f+\delta_{C}+\sum_{t \in T} \lambda_{t} f_{t}\right)\left(x_{0}\right)$. Therefore, (39) holds and the proof is complete.

Corollary 15. Suppose that there exists $x_{0} \in \operatorname{dom} f \cap A$ such that $\partial^{*} f\left(x_{0}\right) \cap\left(-N_{A}\left(x_{0}\right)\right) \neq \emptyset$. Then, for $(P)$ and $(D)$, the total Lagrange duality holds if and only if the strong Lagrange duality holds.

Proof. Let $x^{*} \in \partial^{*} f\left(x_{0}\right) \cap\left(-N_{A}\left(x_{0}\right)\right) \neq \emptyset$. Then, by definition,

$$
\begin{gathered}
\forall x \in X, \quad\left\langle x^{*}, x-x_{0}\right\rangle \geq 0 \Longrightarrow f(x) \geq f\left(x_{0}\right), \\
\left\langle x^{*}, x-x_{0}\right\rangle \geq 0 \quad \text { for each } x \in A .
\end{gathered}
$$

Hence, $x_{0}$ is a minimizer of $f$ on $A$. This implies that $0 \in \partial^{*}\left(f+\delta_{A}\right)\left(x_{0}\right)$. Thus, by Proposition 8 , the quasi(WEHP) and the quasi-(WBCQ) are equivalent. Therefore, by Theorems 9 and 14, the result is seen to hold.

\section{Conflict of Interests}

The authors declare that there is no conflict of interests regarding the publication of this paper. 


\section{Acknowledgments}

Donghui Fang was supported in part by the National Natural Science Foundation of China (Grant 11101186) and supported in part by the Scientific Research Fund of Hunan Provincial Education Department (Grant 13B095). Xianfa Luo was supported in part by the Natural Science Foundation of Zhejiang Province (Grant LY12A01029).

\section{References}

[1] R. I. Boţ and G. Wanka, "Farkas-type results with conjugate functions," SIAM Journal on Optimization, vol. 15, no. 2, pp. 540-554, 2005.

[2] R. I. Boţ, S. M. Grad, and G. Wanka, "On strong and total Lagrange duality for convex optimization problems," Journal of Mathematical Analysis and Applications, vol. 337, no. 2, pp. 1315$1325,2008$.

[3] N. Dinh, M. A. Goberna, and M. A. López, "From linear to convex systems: consistency, Farkas' lemma and applications," Journal of Convex Analysis, vol. 13, no. 1, pp. 279-290, 2006.

[4] N. Dinh, M. A. Goberna, M. A. López, and T. Q. Son, "New Farkas-type constraint qualifications in convex infinite programming," ESAIM Control, Optimisation and Calculus of Variations, vol. 13, no. 3, pp. 580-597, 2007.

[5] D. H. Fang, C. Li, and K. F. Ng, "Constraint qualifications for extended Farkas's lemmas and Lagrangian dualities in convex infinite programming," SIAM Journal on Optimization, vol. 20, no. 3, pp. 1311-1332, 2009.

[6] D. H. Fang, C. Li, and K. F. Ng, "Constraint qualifications for optimality conditions and total Lagrange dualities in convex infinite programming," Nonlinear Analysis: Theory, Methods \& Applications, vol. 73, no. 5, pp. 1143-1159, 2010.

[7] M. A. Goberna, V. Jeyakumar, and M. A. López, "Necessary and sufficient constraint qualifications for solvability of systems of infinite convex inequalities," Nonlinear Analysis: Theory, Methods \& Applications, vol. 68, no. 5, pp. 1184-1194, 2008.

[8] M. A. Goberna and M. A. López, Linear Semi-Infinite Optimization, vol. 2, John Wiley \& Sons, Chichester, UK, 1998.

[9] V. Jeyakumar, "Constraint qualifications characterizing Lagrangian duality in convex optimization," Journal of Optimization Theory and Applications, vol. 136, no. 1, pp. 31-41, 2008.

[10] V. Jeyakumar and H. Mohebi, "Limiting $\epsilon$-subgradient characterizations of constrained best approximation," Journal of Approximation Theory, vol. 135, no. 2, pp. 145-159, 2005.

[11] C. Li, K. F. Ng, and T. K. Pong, "Constraint qualifications for convex inequality systems with applications in constrained optimization," SIAM Journal on Optimization, vol. 19, no. 1, pp. 163$187,2008$.

[12] W. Li, C. Nahak, and I. Singer, "Constraint qualifications for semi-infinite systems of convex inequalities," SIAM Journal on Optimization, vol. 11, no. 1, pp. 31-52, 2000.

[13] H. J. Greenberg and W. P. Pierskalla, "Quasi-conjugate functions and surrogate duality," Cahiers du Centre d'Études de Recherche Opérationnelle, vol. 15, pp. 437-448, 1973.

[14] D. G. Luenberger, “Quasi-convex programming,” SIAM Journal on Applied Mathematics, vol. 16, pp. 1090-1095, 1968.

[15] J. P. Penot and M. Volle, "On quasi-convex duality," Mathematics of Operations Research, vol. 15, no. 4, pp. 597-625, 1990.
[16] S. Suzuki and D. Kuroiwa, "Set containment characterization for quasiconvex programming," Journal of Global Optimization, vol. 45, no. 4, pp. 551-563, 2009.

[17] S. Suzuki and D. Kuroiwa, "Optimality conditions and the basic constraint qualification for quasiconvex programming," Nonlinear Analysis: Theory, Methods \& Applications, vol. 74, no. 4, pp. 1279-1285, 2011.

[18] S. Suzuki and D. Kuroiwa, "On set containment characterization and constraint qualification for quasiconvex programming," Journal of Optimization Theory and Applications, vol. 149, no. 3, pp. 554-563, 2011.

[19] C. Zălinescu, Convex Analysis in General Vector Spaces, World Scientific Publishing, River Edge, NJ, USA, 2002.

[20] P. T. Thach, "Diewert-Crouzeix conjugation for general quasiconvex duality and applications," Journal of Optimization Theory and Applications, vol. 86, no. 3, pp. 719-743, 1995.

[21] J. E. Martínez-Legaz and P. H. Sach, "A new subdifferential in quasiconvex analysis," Journal of Convex Analysis, vol. 6, no. 1, pp. 1-11, 1999. 


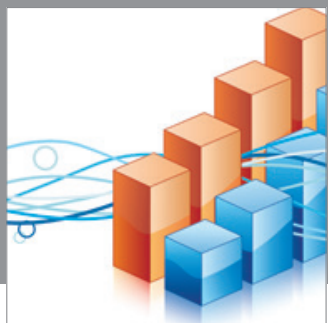

Advances in

Operations Research

mansans

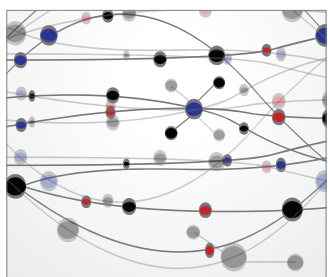

The Scientific World Journal
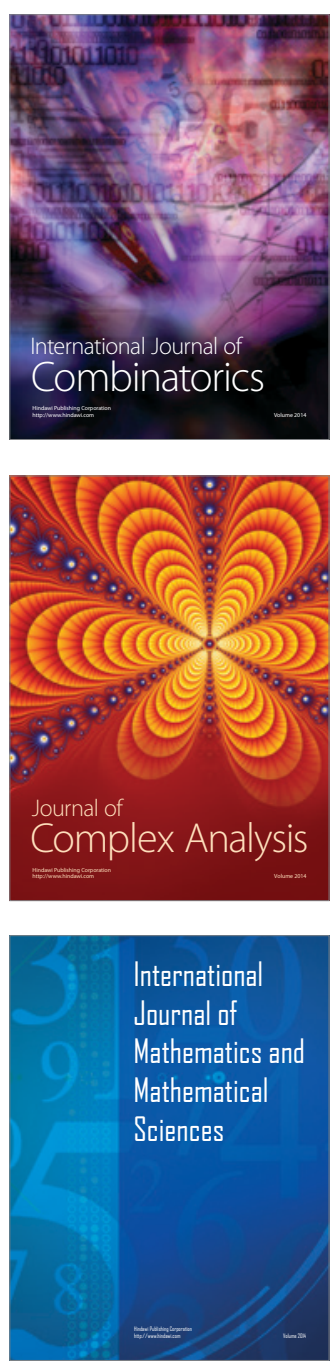
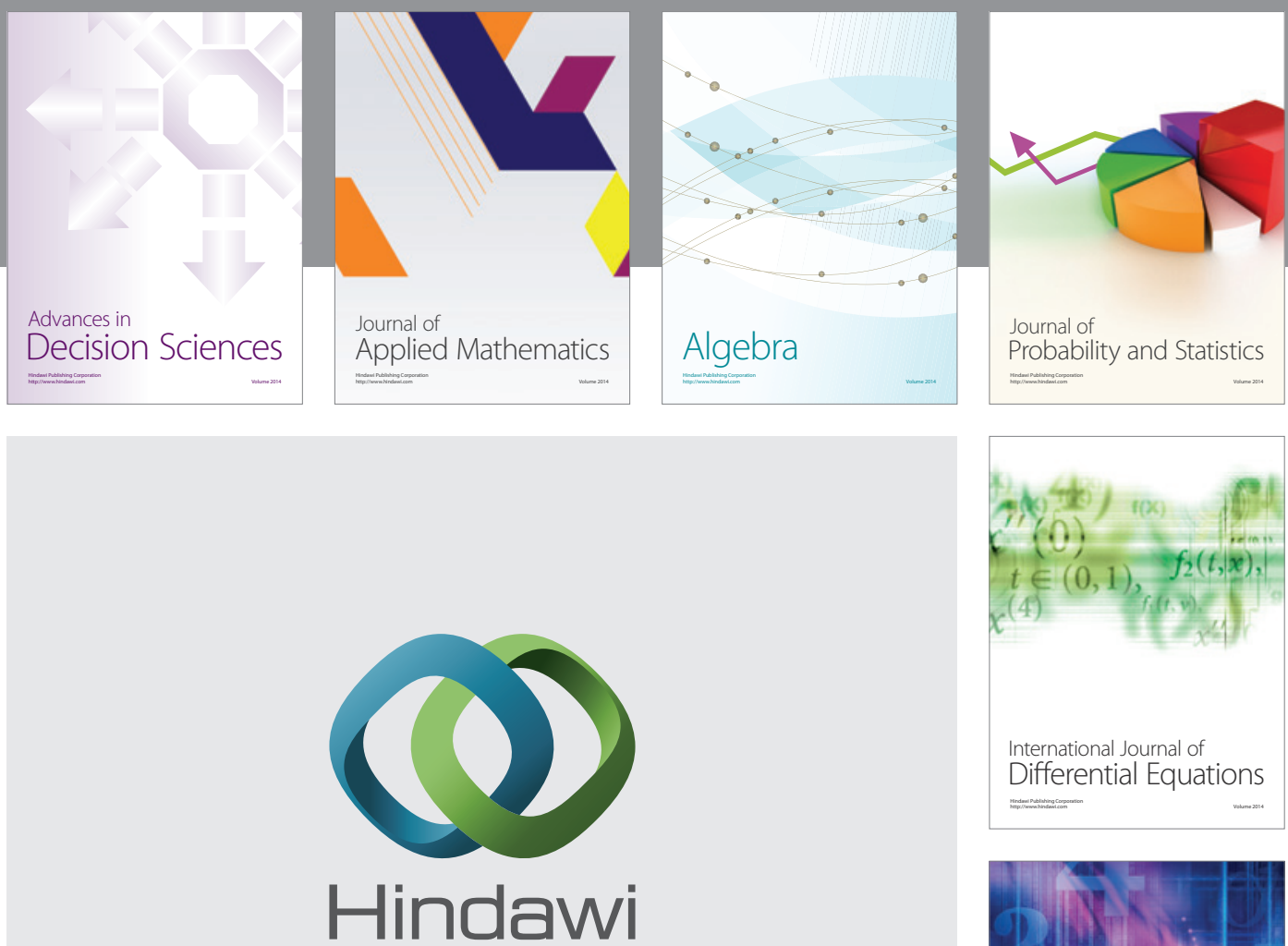

Submit your manuscripts at http://www.hindawi.com
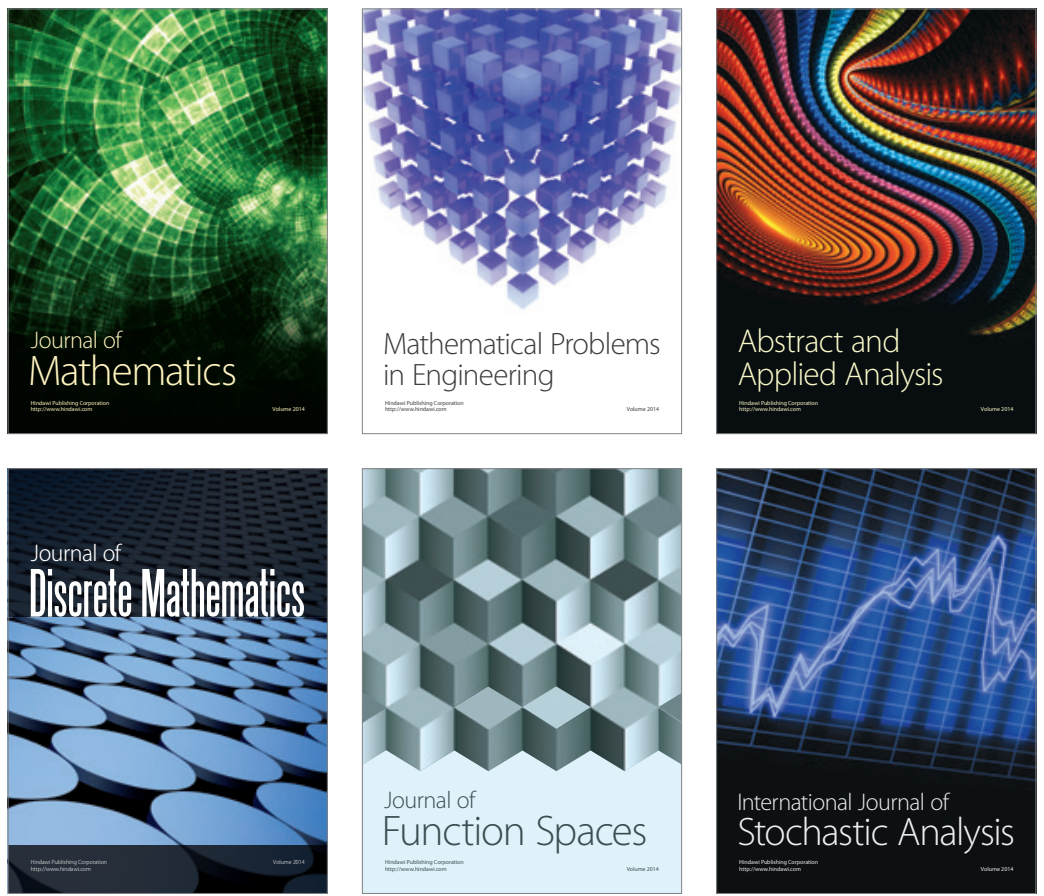

Journal of

Function Spaces

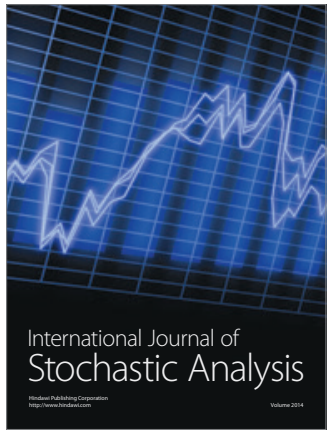

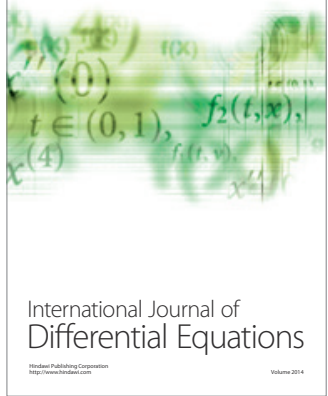
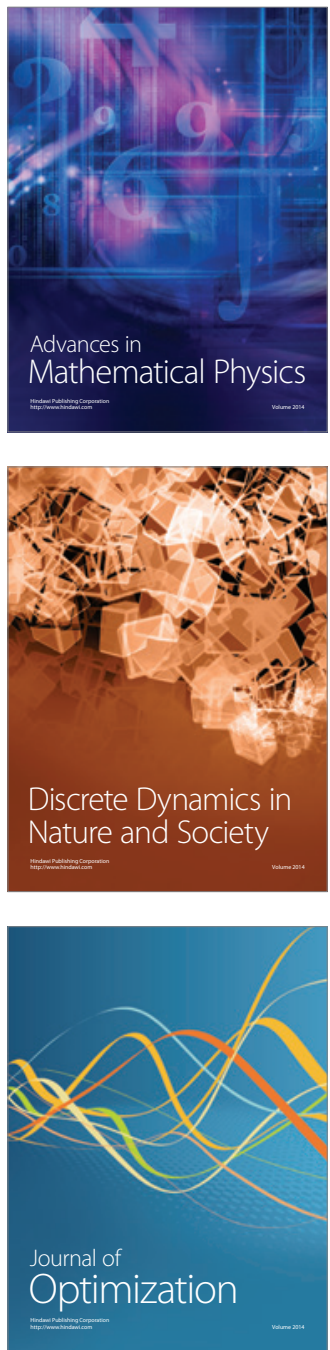